\title{
In vitro Post-Antifungal Effect of Posaconazole and Its Impact on Adhesion-Related Traits and Hemolysin Production of Oral Candida dubliniensis Isolates
}

\author{
Arjuna Nishantha Bandara Ellepola ${ }^{a}$ Ranil Samantha Dassanayake ${ }^{b}$ \\ Ziauddin Khanc \\ ${ }^{a}$ Faculty of Dentistry, Health Sciences Center, Kuwait University, Kuwait, Kuwait; ${ }^{b}$ Faculty of Science, University of \\ Colombo, Colombo, Sri Lanka; ${ }^{C}$ Faculty of Medicine, Health Sciences Center, Kuwait University, Kuwait, Kuwait
}

\section{Significance of the Study}

- The post-antifungal effect, adhesion, and hemolysin production are virulent attributes of Candida isolates.

- Posaconazole is an antifungal agent used in the treatment of candidiasis.

- Posaconazole induced a post-antifungal effect on Candida dubliniensis isolates.

- Posaconazole also suppressed the adhesion attributes and production of hemolysin of Candida dubliniensis isolates.

\section{Keywords}

Adhesion · Candida dubliniensis · Hemolysin ·

Posaconazole $\cdot$ Post-antifungal effect

\begin{abstract}
Objective: Candidal adherence to denture acrylic surfaces (DAS) and oral buccal epithelial cells (BEC), formation of candidal germ tubes (GT), candidal cell surface hydrophobicity (CSH), and hemolysin production are important pathogenic traits of Candida. The antifungal drug-induced post-antifungal effect (PAFE) also impacts the virulence of Candida. Candida dubliniensis isolates are associated with the causation of oral candidiasis which could be managed with posaconazole. Thus far there is no evidence on posaconazole-induced PAFE and its impact on adhesion-related attributes and production of hemolysin by $C$. dubliniensis isolates.
\end{abstract}

Hence, the PAFE, adhesion to DAS and BEC, formation of GT, $\mathrm{CSH}$, and hemolysin production of 20 oral C. dubliniensis isolates after brief exposure to posaconazole was ascertained. Materials and Methods: The PAFE, adherence to DAS and BEC, formation of GT, candidal CSH, and hemolysin production were investigated by hitherto described in vitro assays. Results: The mean PAFE (h) induced by posaconazole on $C$. dubliniensis isolates was 1.66. Exposure to posaconazole suppressed the ability of $C$. dubliniensis to adhere to DAS, BEC, formation of candidal GT, candidal CSH and to produce hemolysin by a reduction of $44,33,34,36$, and $15 \%(p<0.005$ to $p<0.001$ ), respectively. Conclusion: Exposure of $C$. dubliniensis isolates to posaconazole for a brief period induced an antimycotic impact by subduing its growth in addition to suppressing pathogenic adherence-associated attributes, as well as production of hemolysin.

(C) 2019 The Author(s)

Published by S. Karger AG, Basel

\begin{tabular}{ll}
\hline KARGER & $\begin{array}{l}\text { ○ } 2019 \text { The Author(s) } \\
\text { Published by S. Karger AG, Basel Open access }\end{array}$ \\
$\begin{array}{l}\text { E-Mail karger@karger.com } \\
\text { www.karger.com/mpp }\end{array}$ & $\begin{array}{l}\text { This is an Open Access article licensed under the Creative Commons } \\
\text { Attribution-NonCommercial-4.0 International License (CC BY-NC) } \\
\text { (http://www.karger.com/Services/OpenAccessLicense), applicable to } \\
\text { the online version of the article only. Usage and distribution for com- } \\
\text { mercial purposes requires written permission. }\end{array}$
\end{tabular}




\section{Introduction}

In immunologically compromised patients as well as in other patient groups, Candida dubliniensis has been clinically implicated as an important pathogen. For example, in patients infected with human immunodeficiency virus (HIV), this pathogen has been largely associated with oral candidiasis. Moreover, in patients with Candi$d a$-induced denture stomatitis, C. dubliniensis was one of the predominant pathogens recovered [1]. This association between Candida-attributed denture stomatitis suggests an important role for C. dubliniensis in the establishment and persistence of denture stomatitis [1]. This microbe has also been recovered from the oral niches of patients with diabetes and cystic fibrosis patients $[2,3]$. Cases of $C$. dubliniensis fungemia have been reported which is suggestive of dissemination of this species to other ecological niches as well [4]. Furthermore, cases of multifocal osteomyelitis, leptomeningeal disease, spondylodiscitis, and endocarditis have also been linked to $C$. dubliniensis [5-8]. Interestingly, resistance of C. dubliniensis isolates to azoles (i.e., voriconazole, itraconazole, and fluconazole) has also been reported, emphasizing its clinical importance in both oral and systemic candidal infection [9-11].

Post-antifungal effect (PAFE) is the suppression of fungal growth which occurs consequent to brief exposure of fungi to antifungal drugs. Pathogenic traits relating to candidal adherence and Candida enzyme production have also been shown to be modified by limited exposure to drugs with anti-Candida pharmacodynamics [12]. Adhesion of Candida to the host mucosa and oral prosthesis is an important initial factor that facilitates microbial colonization and subsequent candidal infection in the oral cavity [12]. Candida has been shown to attach to host mucous membranes, resulting in Candida-associated mucosal diseases [12]. Therefore, candidal adherence to denture prosthesis and to epithelial cells in the niches of the oral cavity is a vital initial step in the pathogenicity of oral candidal infection $[1,12]$. Additionally, germ tubes (GT) of Candida facilitate Candida adhesion to mucosal cells and even impart resistance to phagocytosis [13]. Hyphal intrusions of Candida stimulate candidal aggregation by bridging adjoining hyphal elements, thereby conveying a large consignment of Candida into intimate interaction with the epithelium [13]. Apart from the above underlying biological forces related to adhesion, cell surface hydrophobicity (CSH) of Candida is a non-biological attribute facilitating adherence of Candida particularly to inert surfaces such as acrylic denture surfaces $[12,14,15]$.
Therefore, Candida which are hydrophobic are believed to be more pathogenic than their hydrophilic counterparts; positive correlations between adherence to denture acrylic surfaces (DAS) and oral buccal epithelial cells (BEC) with CSH of Candida have also been reported [14, 15]. Moreover, in addition to these adhesion-related traits, production of candidal enzymes such as hemolysin also augments the virulent ability of Candida aiding its pathogenesis. Secretion of candidal hemolysin and ensuing lysis of erythrocytes facilitates candidal hyphal invasion $[16,17]$. It also facilitates the release of hemoglobin, which is thereafter utilized by the microbe as a source of iron [17]. Hemoglobin is also an important attribute triggering an alternative channel needed for Candida infections to disseminate in the host [18]. For instance, hemoglobin could facilitate candidal adherence to numerous host proteins and also support Candida to consume exogenous heme or hemoglobin to obtain iron and yield cytoprotective molecules [18]. Such interactions between Candida and hemoglobin seem to be a unique acclimatization of this microbe to be pathogenic in the human host [18].

Posaconazole is a fairly new second-generation azole antifungal drug. It is derived from itraconazole, by replacing the chlorine substituents in the phenyl ring in itraconazole with fluorine and also by hydroxylating the triazolone side chain of itraconazole, which in turn improves the potency and spectrum of activity of posaconazole [19]. Hence, in treating candidal infection caused by $C$. dubliniensis isolates, posaconazole can be used as an alternate drug, particularly with the increased emergence of drug resistance to pharmaceutical agents such as the azoles (i.e., fluconazole, voriconazole, itraconazole) and 5-fluorocytosine (a DNA analogue); where C. dubliniensis isolates resistant to 5-fluorocytosine have been identified in Kuwait and in the Middle-East region [20, 21].

Taking into account the abovementioned background, and the finding of C. dubliniensis oral isolates having the highest prevalence among non-albicans species of Candida in Kuwait [22], the emphasis of the current study was to study the PAFE of posaconazole on oral C. dubliniensis isolates. Furthermore, the effect of this exposure to posaconazole on candidal adherence to DAS and to BEC, candidal GT formation and candidal CSH as well as hemolysin production was also determined. Additionally, as C. dubliniensis are phenotypically similar to Candida albicans, for the purpose of comparison of results between these two species, oral isolates of $C$. albicans acquired from Kuwait were also studied. 


\section{Materials and Methods}

\section{Organisms}

Twenty oral isolates of C. dubliniensis and C. albicans, previously identified and stored as stock cultures were used in the study [22]. These Candida isolates had been previously verified by GT formation and their colony characteristics using CHROMagar Candida medium (Becton Dickinson and Company, Sparks, MD, USA). In addition, profiles of carbohydrate assimilation were obtained using the VITEK 2 Candida identification system (BioMérieux, France). In the same study [22], their identification was further confirmed by means of semi-nested PCR amplification of internally transcribed spacer (ITS)-2 region of rDNA followed by direct DNA sequencing of the ITS region of rDNA. These isolates, obtained from Kuwait, have been used in several previous studies [23-26].

\section{Antifungal Agents and Media}

Posaconazole (Vetranal ${ }^{\circledR}$; Sigma-Aldrich Laborchemikalien $\mathrm{GmbH}$, Darmstadt, Germany) was dissolved in dimethyl sulfoxide. Initially, the drug was made as a solution of $10,000 \mu \mathrm{g} / \mathrm{mL}$ and stored at $-20^{\circ} \mathrm{C}$ [23-26]. Afterwards it was suspended in RPMI 1640 medium containing L-glutamine and lacking sodium bicarbonate, buffered with 0.165 M MOPS (morpholino propanesulfonic acid), dissolved in $1 \mathrm{~L}$ of sterilized distilled water and adjusted to a $\mathrm{pH}$ of 7.2 and filter sterilized.

\section{Determination of Minimum Inhibitory Concentration}

Antifungal susceptibility values for posaconazole for C. albicans and C. dubliniensis isolates were determined by E test according to the manufacturer's recommendations (AB BIODISK, Solna, Sweden) as done in previous studies [23-26]. In brief, each of these Candida isolates were sub-cultured, then five isolated colonies were suspended in sterile saline, and optical density was adjusted to $0.5 \mathrm{McF}$ arland standard to obtain a cell concentration of $1 \times 10^{6}$ to $5 \times 10^{6}$ cells $/ \mathrm{mL}$ as done in previous studies [23-26]. This inoculum of Candida was then swabbed onto $150 \mathrm{~mm}$ diameter plates containing RPMI 1640 agar supplemented with $2 \%$ glucose and buffered with MOPS (0.165 M; pH 7.0) and allowed to dry for 10-15 min. The E test strips were then placed on these agar plates. After incubation for $24-48 \mathrm{~h}$ at $35^{\circ} \mathrm{C}$, the minimum inhibitory concentrations (MIC) were determined. The MIC for each tested isolate was the lowest concentration where the inhibition zone intersected the curve on the posaconazole antifungal strip. C. albicans ATCC 90028 and Candida parapsilosis ATCC 22019 reference strains were used for quality control of the susceptibility testing.

Preparation of Cell Suspension for in vitro Assays (i.e., PAFE, Adhesion to DAS, Adhesion to BEC, GT Formation, Relative $\mathrm{CSH}$, and Hemolysin Production Assays)

Candida cells initially maintained on Sabouraud's dextrose agar (SDA) were freshly inoculated onto SDA plates prior to the experiment and incubated at $37^{\circ} \mathrm{C}$ for $24 \mathrm{~h}$. The resulting Candida were suspended into sterile PBS, and a 1.5 turbidity candidal cell suspension was made. $0.5 \mathrm{~mL}$ of this candidal suspension was poured into tubes containing $2 \mathrm{~mL}$ of RPMI (control) and $2 \mathrm{~mL}$ of RPMI/ posaconazole (test), where the posaconazole concentration was thrice the MIC value. This resulted in $10^{6}$ Candida cells $\mathrm{mL}^{-1}$ suspension in the tubes [23-26]. Subsequently, the tubes were incubated for
$60 \mathrm{~min}$ at $37^{\circ} \mathrm{C}$. Following this limited exposure to the drug, the removal of posaconazole was achieved by three cycles of dilution using sterile PBS followed by centrifugation at $3,000 \mathrm{~g}$ for $10 \mathrm{~min}$. The resulting supernatant was decanted. The Candida pellets were resuspended in $10 \mathrm{~mL}$ of sterile PBS. This procedure has been successfully used in similar studies for the removal of antifungal drugs (i.e., polyenes, azoles, and echinocandins) and has been shown to reduce the drug concentration by 10,000-fold, eliminating any carry-over effect of posaconazole after its removal [23-26]. Viable counts (i.e., colony-forming units) of the test and the control were done following removal of posaconazole, and there was virtually no difference on the viable counts between the test and the control.

\section{PAFE Assay}

After removal of posaconazole, determination of the PAFE was established by an in vitro turbidity method using the equation $\mathrm{PAFE}=\mathrm{T}-\mathrm{C}$ as done in similar PAFE determination studies [2325] ( $\mathrm{T}=$ time for the turbidity of the posaconazole-exposed Candida cell suspension to reach the optical density value of 0.05 at 520 $\mathrm{nm} ; \mathrm{C}=$ time for turbidity of posaconazole-free Candida cell suspension to reach the optical density value of 0.05 at $520 \mathrm{~nm}$ ). Thus, $\mathrm{T}-\mathrm{C}$ indicates the duration by which posaconazole was able to suppress candidal growth following brief exposure to posaconazole (i.e., PAFE). For this purpose, as done in previous investigations [23-25], 1,600 $\mu \mathrm{L}$ of Candida cell suspensions were incubated at $37^{\circ} \mathrm{C}$ after mixing with $2.4 \mathrm{~mL}$ of RPMI 1640, and the turbidity of this mixture was recorded every $15 \mathrm{~min}$ for $6 \mathrm{~h}$; during this period both the test and control suspensions arrived at the optical density of 0.05 , permitting the calculation of PAFE induced by posaconazole.

\section{Adherence to DAS}

For the adherence assay, acrylic strips were made as reported previously $[15,26]$. Polymethyl methacrylate powder was spread over the surface of a $2.5 \times 7.5 \mathrm{~cm}$ glass slide covered with a thin layer of aluminum foil. Onto this aluminum foil $1 \mathrm{~mL}$ of monomer liquid was poured and immediately another slide was placed over the polymerizing mixture. Using two binder clips, the two slides were then firmly clasped at both ends. After allowing to bench cure for $30 \mathrm{~min}$, the binder clips were removed and the glass slides were separated. The resulting acrylic strips were cut into squares of $5 \times$ $5 \mathrm{~mm}$ and were submerged in sterile distilled water for 1 week to allow excess monomer to seep out, and then washed in running water for $3 \mathrm{~h}$. These acrylic strips were then disinfected by immersing in $70 \%$ alcohol and washed with sterile distilled water. To remove any contaminants from the surfaces, the acrylic strips were subjected to ultra-sonication for $20 \mathrm{~min}$ and rewashed in sterile distilled water and then dried.

The strips were placed vertically and kept in wells of sterilized, serological plates. $400 \mu \mathrm{L}$ of candidal suspension was added to each well submerging the strip within the well. These plates were then incubated at $37^{\circ} \mathrm{C}$ for $1 \mathrm{~h}$ with agitation at 120 revolutions/min. The strips were then washed thrice by dipping gently in sterile PBS, and then dried and stained with modified Gram stain without the counterstain. These strips were allowed to air-dry at room temperature and mounted on glass slides with glycerol and the attached Candida counted under a light microscope $(\times 400$ magnification). Adherent Candida cells in 20 fields of view for each strip $\left(0.25 \mathrm{~mm}^{2}\right.$ per field) were counted, and the counts were expressed as Candida cells $/ \mathrm{mm}^{2}$. 


\section{BEC Adherence}

Human BEC from 5 adults were acquired by gently rubbing the inner surface of their right and left buccal mucosa with sterile cotton swabs. This pool of human BEC were then added into tubes containing sterilized PBS. The pooled BEC suspension was washed in sterile PBS to detach any microbes and centrifuged for $10 \mathrm{~min}$ at $3,500 \mathrm{~g}$. The resulting pellets of BEC (devoid of any microbes) were mixed with sterile PBS to obtain a BEC concentration of $1 \times$ $10^{5}$ cells $/ \mathrm{mL}$. For the adherence assay, $0.5 \mathrm{~mL}$ of Candida suspension (previously unexposed to posaconazole and briefly exposed to the drug) and $0.5 \mathrm{~mL}$ of the BEC suspension were mixed in sterilized plastic tubes and incubated for $1 \mathrm{~h}$ at $37^{\circ} \mathrm{C}$ to facilitate candidal adherence to BEC. The resulting suspension was then diluted using $4 \mathrm{~mL}$ of sterile PBS. Thereafter, BEC were gathered onto polycarbonate filters ( $12 \mu \mathrm{m}$ pore size). These polycarbonate filters were subjected to a gentle wash with sterile PBS to remove any Candida not attached to BEC. The filters were then positioned on a glass slide. After a period of $15 \mathrm{~s}$, the filters were removed. The resulting cell preparations on these slides were dried and Gramstained. Thereafter, using light microscopy ( $\times 400$ magnification), the adherent Candida cells were quantified. BEC in 50 successive fields were observed to count the Candida cells adhered to BEC.

\section{Quantification of Candidal GT-Forming Cells}

For generation of candidal GT formation, a suspension of 250 $\mu \mathrm{L}$ of Candida (recovered following posaconazole removal and the unexposed control), was added to $1 \mathrm{~mL}$ of RPMI 1640 medium with L-glutamine and incubated for $90 \mathrm{~min}$ at $37^{\circ} \mathrm{C}$. Thereafter, these tubes were vortex mixed for $10 \mathrm{~s}$ and a drop of each cell suspension was placed on a Neubauer's hemocytometer chamber and covered with a cover slip for enumeration of GT formed. $300 \mathrm{Can}$ dida cells in adjacent fields were counted under $\times 40$ magnification and the percentage of Candida cells forming GT was estimated. Only Candida cells with a GT, with no constriction at the junction between the cell and the elongation were counted. Clumped Candida cells and pseudo-hyphae-forming Candida cells were omitted $[24,25]$.

\section{Candidal CSH Assay}

$2.5 \mathrm{~mL}$ of a Candida cell suspension (posaconazole exposed as well as posaconazole-free controls) was poured into $20 \mathrm{~mL}$ sterilized glass test tubes, after which $0.5 \mathrm{~mL}$ of xylene was added into these glass tubes. These tubes were incubated for $10 \mathrm{~min}$ at $37^{\circ} \mathrm{C}$ to allow the two layers to equilibrate, and then vortex-mixed for 30 $s$ and incubated for an additional $30 \mathrm{~min}$ to allow the xylene layer to become distinct from the aqueous phase. The aqueous phase at the bottom of the tube was aspirated using a pipette and put into a sterilized test tube. Traces of xylene were eliminated by bubbling air through the suspension for $2 \mathrm{~min}$. After vortex-mixing for $5 \mathrm{~s}$, the turbidity of this mixture was measured at $520 \mathrm{~nm}$. Candidal $\mathrm{CSH}$ was calculated as the reduction in percentage in the optical density value of the later suspension compared to the initial suspension (i.e., before adding xylene) $[14,15,24,25]$.

\section{Hemolysin Production Assay}

$15 \mu \mathrm{L}$ of the aforementioned posaconazole-exposed and -unexposed suspensions were spot-inoculated onto blood agar enriched with $3 \%$ glucose, which resulted in a circular inoculation site of approximately $10 \mathrm{~mm}$ in diameter. The plates were then incubated at $37^{\circ} \mathrm{C}$ in $5 \% \mathrm{CO}_{2}$ for a period of $48 \mathrm{~h}$. The diameter of the colony
Table 1. The PAFE (in hours) of 20 oral C. dubliniensis and C. albicans isolates following 1-hour exposure to posaconazole

\begin{tabular}{llll}
\hline $\begin{array}{l}\text { C. dubliniensis } \\
\text { isolate }\end{array}$ & PAFE & $\begin{array}{l}\text { C. albicans } \\
\text { isolate }\end{array}$ & PAFE \\
\hline CD1 & 2.26 & CA1 & 2.12 \\
CD2 & 1.26 & CA2 & 2.12 \\
CD3 & 1.62 & CA3 & 1.26 \\
CD4 & 1.62 & CA4 & 1.41 \\
CD5 & 1.41 & CA5 & 1.26 \\
CD6 & 1.62 & CA6 & 1.41 \\
CD7 & 1.47 & CA7 & 1.47 \\
CD8 & 2.12 & CA8 & 2.12 \\
CD9 & 2.39 & CA9 & 2.12 \\
CD10 & 2.33 & CA10 & 2.12 \\
CD11 & 1.47 & CA11 & 1.26 \\
CD12 & 1.41 & CA12 & 1.41 \\
CD13 & 1.26 & CA13 & 1.26 \\
CD14 & 1.26 & CA14 & 1.41 \\
CD15 & 1.26 & CA15 & 1.26 \\
CD16 & 1.62 & CA16 & 1.47 \\
CD17 & 1.62 & CA17 & 1.47 \\
CD18 & 1.41 & CA18 & 1.41 \\
CD19 & 2.12 & CA19 & 2.12 \\
CD20 & 1.62 & CA20 & 1.47 \\
\hline Mean & 1.66 & Mean & 1.60 \\
SEM & 0.08 & SEM & 0.08 \\
\hline
\end{tabular}

In calculating the PAFE, $1=60 \mathrm{~min}, 0.25=15 \mathrm{~min}, 0.50=30$ $\min$, and $0.75=45 \mathrm{~min}$ in the table. Hence, for isolate CD1 the PAFE of 2.26 equals $2 \mathrm{~h}$ and $15 \mathrm{~min}$. All values indicate the mean of experiments done thrice in duplicate for each isolate.

(y) and the diameter of the colony plus the translucent zone due to hemolysis ( $\mathrm{x}$ ) were measured. The hemolytic activity (hemolytic index), which is the ratio of $\mathrm{x} / \mathrm{y}$, was determined as reported previously $[16,23,25]$.

C. dubliniensis reference strain CD36 and C. albicans reference strain ATCC 90028 were included in all the abovementioned experiments. All experiments were repeated three times in duplicate.

\section{Statistical Analysis}

The data obtained from adherence to DAS, adherence to BEC, formation of GT, candidal CSH, and hemolysin assays were analyzed using paired sample $t$ tests, with the group unexposed to posaconazole as the control and the group exposed to posaconazole as the test group. A $p$ value of $<0.05$ was considered statistically significant.

\section{Results}

The MIC $(\mu \mathrm{g} / \mathrm{mL})$ of $C$. dubliniensis to posaconazole varied between 0.002 and 0.016 . The mean PAFE on these isolates after a 1-h exposure to posaconazole was $1.66 \mathrm{~h}$. 
Table 2. Summary of adherence to BEC (Candida cells/50 BEC), adherence to DAS (Candida cells $/ \mathrm{mm}^{2}$ ), mean percentage of GT-positive cells, candidal CSH, and hemolysin production (hemolytic index) of 20 oral C. dubliniensis and C. albicans isolates following brief exposure and subsequent removal of posaconazole

\begin{tabular}{lccc}
\hline Pathogenic attribute & $\begin{array}{c}\text { Control (unexposed } \\
\text { to posaconazole) }\end{array}$ & $\begin{array}{l}\text { Test (exposed } \\
\text { to posaconazole) }\end{array}$ & $\begin{array}{l}\text { Mean percentage } \\
\text { reduction and significance }\end{array}$ \\
\hline C. dubliniensis isolates & & & \\
Adherence to BEC & $203.24 \pm 2.78$ & $136.67 \pm 2.58$ & $32.76 \% ; p<0.001$ \\
Adherence to DAS & $45.06 \pm 0.58$ & $25.14 \pm 0.53$ & $44.21 \% ; p<0.001$ \\
GT formation & $24.74 \pm 0.61$ & $16.38 \pm 0.40$ & $33.79 \% ; p<0.001$ \\
Candidal CSH & $17.54 \pm 0.20$ & $11.30 \pm 0.13$ & $35.58 \% ; p<0.001$ \\
Hemolysin production & $1.60 \pm 0.008$ & $1.36 \pm 0.008$ & $15.00 \% ; p<0.005$ \\
C. albicans isolates & & & \\
Adherence to BEC & $205.32 \pm 2.14$ & $143.18 \pm 2.41$ & $30.26 \% ; p<0.001$ \\
Adherence to DAS & $46.74 \pm 0.76$ & $26.44 \pm 0.57$ & $43.44 \% ; p<0.001$ \\
GT formation & $26.03 \pm 0.81$ & $17.38 \pm 0.58$ & $33.25 \% ; p<0.001$ \\
Candidal CSH & $16.96 \pm 0.39$ & $11.37 \pm 0.21$ & $32.96 \% ; p<0.001$ \\
Hemolysin production & $1.61 \pm 0.007$ & $1.41 \pm 0.007$ & $12.42 \% ; p<0.005$ \\
\hline
\end{tabular}

All values indicate the mean of experiments done thrice in duplicate for each isolate.

The MIC $(\mu \mathrm{g} / \mathrm{mL})$ of C. albicans to posaconazole ranged from 0.012 to 0.016 . The posaconazole-induced mean PAFE on oral isolates of C. albicans was $1.60 \mathrm{~h}$ (Table 1).

The mean adherence of $C$. dubliniensis to DAS (cells/ $\mathrm{mm}^{2}$ ) not exposed to posaconazole and following limited exposure to the drug was 45.06 and 25.14 , respectively (44.21\% reduction; $p<0.001)$. Likewise, these values for C. albicans isolates not exposed to posaconazole and after limited exposure to posaconazole were 46.74 and 26.44 , respectively (43.44\% reduction; $p<0.001$; Table 2 ).

The mean adherence of $C$. dubliniensis isolates to BEC not exposed to posaconazole and following brief acquaintance to posaconazole was 203.24 and 136.67 , respectively $(32.76 \%$ reduction; $p<0.001)$. Similarly, these values for isolates of $C$. albicans not exposed to posaconazole and after brief exposure to posaconazole were 205.32 and 143.18 , respectively $(30.26 \%$ reduction; $p<0.001$; Table 2).

The mean percentages of cells with GT not exposed to posaconazole and after transient exposure to this drug were 24.74 and 16.38 , respectively (reduction of $33.80 \%$; $p<0.001)$. These assessments for C. albicans isolates unexposed to posaconazole and following brief exposure to posaconazole were 26.03 and 17.38 , respectively (reduction of $33.25 \%$; $p<0.001$; Table 2).

The mean CSH of C. dubliniensis isolates not exposed to posaconazole and after limited acquaintance to this drug was 17.54 and 11.30 , respectively (reduction of $35.58 \% ; p<0.001$ ). These values for isolates of C. albicans not exposed to posaconazole and after limited acquain- tance to posaconazole were 16.96 and 11.37 , respectively (reduction of $32.96 \% ; p<0.001$; Table 2).

Mean hemolysin production by unexposed C. dubliniensis isolates was 1.60. After brief exposure to posaconazole it was 1.36 (reduction of $15.00 \% ; p<0.005$ ). Likewise, the mean hemolytic index by unexposed C. albicans isolates was 1.61, whereas following brief exposure to posaconazole it was 1.41 (reduction of $12.42 \% ; p<0.005$; Table 2).

\section{Discussion}

C. dubliniensis is an opportunistic yeast implicated in oral candidiasis, while C. albicans is considered to be the most pathogenic of all Candida species responsible for oral candidiasis [12]. Adhesion of Candida to DAS and to $\mathrm{BEC}$ of the oral mucosa and the ability of $C$. dubliniensis and C. albicans isolates to produce GT are critical pathogenic attributes relating to their adherence, which are closely allied with all forms of oral candidiasis $[1,12,13]$. In addition to these biological adherence traits, candidal $\mathrm{CSH}$ is considered to be a non-biological physical attribute believed to facilitate adhesion of Candida $[14,15]$. Another factor determining Candida virulence is the PAFE, which is the capability of Candida to recover and grow, following brief exposure to antifungal agents, where more susceptible and less virulent Candida will have a higher PAFE, whereas more resilient and pathogenic Candida will have a lower PAFE [12, 23, 24, 26]. 
The findings in this study reveal that posaconazole brought about a PAFE of nearly $1 \frac{112}{2}$ on both Candida species investigated following 1-h exposure to posaconazole. This study also found a marked reduction in adherence to DAS and BEC, and formation of candidal GT of both C. albicans and C. dubliniensis oral isolates. These suppressive effects on growth and adhesion could be attributed to the mechanism of action of posaconazole on the candidal cell wall. Posaconazole inhibits fungal cytochrome P450-mediated 14-alpha lanosterol demethylation, which in turn blocks ergosterol synthesis [27]. The lack of ergosterol reduces the stability of the candidal cell membrane, the transport of nutrients, and the synthesis of chitin [28]. In addition, ergosterol depletion accompanied by a buildup of toxic methylated ergosterol precursors will bring about damage to the fungal cell membrane, increased permeability, and inhibition of fungal cell growth $[29,30]$. Thus, it is reasonable to speculate that posaconazole-induced alterations in the structure of the Candida cell wall could disturb active multiplication and budding of Candida, thus attenuating candidal growth and resulting in a PAFE of approximately $1 \frac{1}{2} \mathrm{~h}$, besides limiting candidal adherence to DAS and BEC, and formation of GT even after a limited exposure to posaconazole.

Phospholipids, lipopolysaccharides, proteins, lipoproteins, and fimbriae of the outer membrane are structures that augment microbial CSH $[14,15]$. Thus, pharmaceutical agents which could modify these structures of microbes have been shown to reduce microbial CSH [23, 26]. In Candida in particular, it has been shown that candidal CSH correlates well with the fibril concentration in the exterior layer of the cell wall. Hence, the apparent decrease of CSH elicited by posaconazole on isolates of $C$. albicans and C. dubliniensis may also be attributed to the aforesaid mechanism of action of posaconazole on the cell wall of these two species $[27,30]$. Hence, it is reasonable to suggest that by debilitating the candidal cell wall structure posaconazole could bring about a significant decrease in the CSH of the two Candida species.

Another well-recognized, putative virulent factor of Candida is the production of extracellular hemolysin [17, 18]. This study illustrates that exposure to posaconazole succeeds in suppressing hemolysin production of both C. albicans and C. dubliniensis isolates to a significant level. However, the precise pharmacodynamics by which posaconazole reduces the production of hemolysin by these two Candida species is not clear. Posaconazole acts by inhibiting fungal cytochrome $\mathrm{P} 450$-mediated 14-alpha lanosterol demethylation, which in turn blocks ergosterol synthesis, thus affecting the integrity of candidal cell wall
[27-30]. This may have an impact on hemolytic activity following exposure to posaconazole. Interestingly, hemolysin is also known to facilitate host invasion by Candida, particularly by aiding hyphal penetration of the mucosa. Thus, posaconazole could curtail dissemination of Candida within the host by reducing hemolysin production and formation of candidal GT as well. As disseminated Candida infections are becoming quite common [4-8, 12], posaconazole may have a negative impact on disease progression by reducing the aforesaid pathogenic attributes of C. dubliniensis and C. albicans.

Resistance to itraconazole, voriconazole, and fluconazole has been recognized previously in C. dubliniensis [9-11]. Resistance to 5 -fluorocytosine has been recently documented in isolates obtained from the Middle-East region and Kuwait $[20,21]$. Such candidal resistance signifies the need for identifying promising alternative antifungal agents, which may assist the management of candidiasis. These preliminary findings suggest that brief exposure to posaconazole induces a PAFE and also suppresses adherence to DAS and BEC as well as GT formation, relative CSH, and hemolysin production. This may further contribute to understanding the pharmacodynamics of posaconazole.

\section{Conclusion}

To the best of our knowledge, this investigation is the first to reveal the reduction of adhesion, hemolysin production, and the PAFE induced by posaconazole on oral C. dubliniensis and C. albicans isolates. However, further studies with sessile Candida isolates encased within biofilms, in contrast to planktonic isolates utilized in the present investigation, are needed to further enhance these in vitro findings.

\section{Acknowledgements}

This work was supported and funded by Kuwait University Research Grant No. DB 01/17. The technical support and advice from Ms. Rachel Chandy, former chief technician, Department of Microbiology, Faculty of Medicine, Kuwait University, Kuwait, is thankfully acknowledged. The valuable contribution of Dr. Jagan Kumar, Faculty of Dentistry, Kuwait University, Kuwait, in statistical analysis of data is much appreciated.

\section{Disclosure Statement}

The authors declare that there is no conflict of interest in relation to this study. 


\section{References}

1 Zomorodian K, Haghighi NN, Rajaee N, Pakshir K, Tarazooie B, Vojdani M, et al. Assessment of Candida species colonization and denture-related stomatitis in complete denture wearers. Med Mycol. 2011 Feb;49(2): 208-11.

2 Peltroche-Llacsahuanga $H$, Döhmen $H$, Haase G. Recovery of Candida dubliniensis from sputum of cystic fibrosis patients. Mycoses. 2002 Feb;45(1-2):15-8.

3 Manfredi M, McCullough MJ, Al-Karaawi ZM, Hurel SJ, Porter SR. The isolation, identification and molecular analysis of Candida spp. isolated from the oral cavities of patients with diabetes mellitus. Oral Microbiol Immunol. 2002 Jun;17(3):181-5.

4 Brandt ME, Harrison LH, Pass M, Sofair AN, Huie S, Li RK, et al. Candida dubliniensis fungemia: the first four cases in North America. Emerg Infect Dis. 2000 Jan-Feb;6(1): 46-9.

5 Andrew NH, Ruberu RP, Gabb G. The first documented case of Candida dubliniensis leptomeningeal disease in an immunocompetent host. BMJ Case Rep. 2011 Aug;2011: bcr0620114384.

6 Garcia J, Soch K, Matthew E, Surani S, Horseman MA. Endocarditis caused by Candida dubliniensis. Am J Med Sci. 2013 Sep;346(3): 237-9.

7 Wellinghausen N, Moericke A, Bundschuh S, Friedrich W, Schulz AS, Gatz SA. Multifocal osteomyelitis caused by Candida dubliniensis. J Med Microbiol. 2009 Mar;58(Pt 3):38690.

8 Oksi J, Finnilä T, Hohenthal U, RantakokkoJalava K. Candida dubliniensis spondylodiscitis in an immunocompetent patient. Case report and review of the literature. Med Mycol Case Rep. 2013 Nov;3:4-7.

9 Fanci R. Breakthrough Candida dubliniensis fungemia in an acute myeloid leukemia patient during voriconazole therapy successfully treated with caspofungin. J Chemother. 2009 Feb;21(1):105-7.

10 Moran GP, Sullivan DJ, Henman MC, McCreary CE, Harrington BJ, Shanley DB, et al. Antifungal drug susceptibilities of oral Candida dubliniensis isolates from human immunodeficiency virus (HIV)-infected and non-
HIV-infected subjects and generation of stable fluconazole-resistant derivatives in vitro. Antimicrob Agents Chemother. 1997 Mar; 41(3):617-23.

11 Fleischhacker M, Pasligh J, Moran G, Ruhnke $M$. Longitudinal genotyping of Candida dubliniensis isolates reveals strain maintenance, microevolution, and the emergence of itraconazole resistance. J Clin Microbiol. 2010 May;48(5):1643-50.

12 Ellepola AN, Samaranayake LP. Oral candidal infections and antimycotics. Crit Rev Oral Biol Med. 2000;11(2):172-98.

13 Tronchin G, Bouchara JP, Robert R, Senet JM Adherence of Candida albicans germ tubes to plastic: ultrastructural and molecular studies of fibrillar adhesins. Infect Immun. 1988 Aug; 56(8):1987-93.

14 Panagoda GJ, Ellepola AN, Samaranayake LP. Adhesion to denture acrylic surfaces and relative cell-surface hydrophobicity of Candida parapsilosis and Candida albicans. APMIS. 1998 Jul;106(7):736-42.

15 Panagoda GJ, Samaranayake LP. The relationship between the cell length, adhesion to acrylic and relative cell surface hydrophobicity of Candida parapsilosis. Med Mycol. 1998 Dec;36(6):373-8.

16 Luo G, Samaranayake LP, Yau JY. Candida species exhibit differential in vitro hemolytic activities. J Clin Microbiol. 2001 Aug;39(8): 2971-4.

17 Manns JM, Mosser DM, Buckley HR. Production of a hemolytic factor by Candida albicans. Infect Immun. 1994 Nov;62(11):5154-6.

18 Pendrak ML, Yan SS, Roberts DD. Sensing the host environment: recognition of hemoglobin by the pathogenic yeast Candida albicans. Arch Biochem Biophys. 2004 Jun;426(2): $148-56$.

19 Soysal A. Prevention of invasive fungal infections in immunocompromised patients: the role of delayed-release posaconazole. Infect Drug Resist. 2015 Sep;8:321-31.

20 Al Mosaid A, Sullivan DJ, Polacheck I, Shaheen FA, Soliman O, Al Hedaithy S, et al. Novel 5-Flucytosine-Resistant Clade of Candida dubliniensis from Saudi Arabia and Egypt Identified by Cd25 Fingerprinting. J Clin Microbiol. 2005;43:4026-36.
21 Ahmad S, Khan ZU, Joseph L, Asadzadeh M, Theyyathel A. Genotypic heterogeneity and molecular basis of 5-flucytosine resistance among Candida dubliniensis isolates recovered from clinical specimens in Kuwait. Med Mycol. 2012 Apr;50(3):244-51.

22 Ellepola AN, Khan ZU, Joseph B, Chandy R, Philip L. Prevalence of Candida dubliniensis among oral Candida isolates in patients attending the Kuwait University Dental Clinic. Med Princ Pract. 2011;20(3):271-6.

23 Ellepola AN, Khajah R, Jayatilake S, Samaranayake L, Sharma P, Khan Z. Impact of brief exposure to antifungal agents on the post-antifungal effect and hemolysin activity of oral Candida albicans. J Appl Oral Sci. 2015 JulAug;23(4):412-8.

24 Ellepola AN, Joseph BK, Chandy R, Khan ZU. The postantifungal effect of nystatin and its impact on adhesion attributes of oral Candida dubliniensis isolates. Mycoses. 2014 Jan; 57(1):56-63.

25 Ellepola AN, Chandy R, Khan ZU. In vitro postantifungal effect, adhesion traits and haemolysin production of Candida dubliniensis isolates following exposure to 5-fluorocytosine. Mycoses. 2015 Sep;58(9):536-43.

26 Ellepola AN, Joseph BK, Altarakemah Y, Samaranayake LP, Anil S, Hashem M, et al. In vitro adhesion of oral Candida dubliniensis isolates to acrylic denture surfaces following brief exposure to sub-cidal concentrations of polyenes, azoles and chlorhexidine. Med Princ Pract. 2015;24(1):58-64.

27 Katragkou A, Tsikopoulou F, Roilides E, Zaoutis TE. Posaconazole: when and how? The clinician's view. Mycoses. 2012 Mar;55(2): $110-22$

28 Hof H. A new, broad-spectrum azole antifungal: posaconazole-mechanisms of action and resistance, spectrum of activity. Mycoses. 2006;49(s1 Suppl 1):2-6.

29 Torres HA, Hachem RY, Chemaly RF, Kontoyiannis DP, Raad II. Posaconazole: a broadspectrum triazole antifungal. Lancet Infect Dis. 2005 Dec;5(12):775-85.

30 Kwon DS, Mylonakis E. Posaconazole: a new broad-spectrum antifungal agent. Expert Opin Pharmacother. 2007 Jun;8(8): 1167-78. 